\title{
Nonexponential dielectric relaxation dynamics in supercooled liquid and glassy states of isoamyl bromide and 2-methylpentane mixtures
}

\author{
O. E. Kalinovskaya and J. K. Vij ${ }^{\mathrm{a})}$ \\ Laboratory of Advanced Materials, Department of Electronic and Electrical Engineering, \\ Trinity College, University of Dublin, Dublin 2, Ireland
}

(Received 25 May 2000; accepted 14 November 2000)

\begin{abstract}
The dielectric spectra of mixtures of the polar solute isoamyl bromide in 2-methylpentane have been investigated in the frequency range $1 \mathrm{mHz}$ to $1 \mathrm{MHz}$ and in the temperature range approaching the glass transition temperature. The results obtained from the spectra are compared with those obtained recently [J. Chem. Phys. 111, 10979 (1999)] on pure isoamyl bromide. It is found that on increasing dilution with the nonpolar solvent, the width of the curves of the dielectric spectra increase significantly, and this is reflected in the increase in the nonexponential nature of the relaxation dynamics. This is found to be a consequence of the decrease in the cooperativity of the relaxation dynamics and or an increase in the heterogeneity of the solution. The data are found to fit the Havriliak-Negami equation extremely well. The data at low and high frequencies also fits the "universal law," since the latter is a low and high frequencies limiting case of the HavriliakNegami equation. The scaling parameters of this law are calculated for the $25 \mathrm{~mol} \%$ solution of isoamyl bromide in 2-methylpentane, and these are shown to experimentally relate to the $\mathrm{H}-\mathrm{N}$ parameters. The stretched exponential parameter, $\gamma$, is estimated as a function of the temperature and is shown to follow the equation $\gamma \approx a\left(T-T_{0}\right)$. Vogel-Fulcher-Tammann equation fits the data of the relaxation peak frequency as a function of the inverse of absolute temperature for the various mixtures quite well, this being possibly a consequence of the temperature dependence of the stretched exponential parameter. The predictions from the mode coupling theory and those by Bertrand and Souletie are verified with the exception that the exponent is found to be much greater than predicted by these theories. The relative predominance of the Johari-Goldstein process in isoamyl bromide increases initially with dilution with 2-methylpentane and then disappears as the number density of the independent relaxors increases with further dilution. (C) 2001 American Institute of Physics. [DOI: 10.1063/1.1338511]
\end{abstract}

\section{INTRODUCTION}

Molecular dynamics of glass-forming liquids not only has crucial ramifications for the fundamental study of liquids, but also has the important significance for glass and polymer science. Liquids that supercool, rather than freeze, simulate the behavior of polar and nonpolar polymers with a well defined glass transition temperature. Dielectric spectroscopy is a very powerful technique for investigating the molecular dynamics of supercooled liquids due to the availability of an extended range of frequencies of usually 15 decades or wider. This is nearly half of the entire conceivable range of frequencies in the universe, which extend from frequencies of the cosmic radiation of $10^{25} \mathrm{~s}^{-1}$ to the reciprocal age of the universe of $10^{-15} \mathrm{~s}^{-1}$, a total of 40 decades of frequency. The useful range of frequencies however where the relaxation phenomena due to the intermolecular interactions may dominate is at the most about 18 decades wide, and the dielectric spectroscopy can cover most of it.

In this paper, we report the dielectric spectra of isoamyl bromide (1-bromo-3 methyl butane) in solutions of 2-methylpentane and compare with the spectra of pure isoamyl bromide reported in our previous paper. ${ }^{1}$ A skewed

\footnotetext{
a) Author to whom correspondence should be addressed. Electronic mail: jvij@tcd.ie
}

arc shape in the complex permittivity plots was observed for pure isoamyl bromide above its freezing point by Glarum, ${ }^{2}$ by Denny ${ }^{3}$ in the supercooled state, and by Berberian and Cole $^{4}$ and Berberian ${ }^{5}$ who later extended these measurements to lower temperatures. The dielectric relaxation of its solution over a limited range of temperatures and frequencies was studied by Denny and Ring. ${ }^{6}$ The solute isoamyl bromide is selected because it is a simple polar liquid devoid of hydrogen-bonding. The solvent hydrocarbon 2-methylpentane is chosen because (i) isoamyl bromide completely dissolves in it, (ii) it does not hydrogen-bond, and (iii) the molecules of these two liquids are isometric, i.e., they have the same size and shape, except that one is polar and the other nonpolar. The molecular shape is the same for the reason that the bromine group is nearly of the same size as the methyl group. These liquids and their mixtures are known to supercool without freezing. In our previous paper, ${ }^{1}$ we found that the relaxation mechanism for pure isoamyl bromide follows the Davidson-Cole ${ }^{7}$ formalism, similar to the conclusions of Berberian and Cole, ${ }^{4}$ whereas that of its $50 \mathrm{~mol} \%$ mixture surprisingly showed a remarkable change in the spectra and followed the Havriliak-Negami ${ }^{8}$ function with both parameters much different than unity. Mixtures of isoamyl bromide in 2-methylpentane are the ideal systems to study the nonexponential character of the relaxation func- 
tion. In this paper we extend these measurements to 25 and $75 \mathrm{~mol} \%$ solutions of isoamyl bromide in 2-methylpentane. The objectives of the study are to determine (i) the effect on the dielectric spectra of varying the concentration of a simple nonhydrogen bonded liquid in a solvent whose molecules are of similar shape, (ii) how a change in the electrostatic interactions of a dipolar molecule in a nonpolar solvent of similar type affect the shape and the parameters of the relaxation process which is largely nonexponential, and (iii) to find the dependence of the time-domain stretched exponential parameter, a measure of the nonexponential character of the relaxation function, on the temperature as the system is supercooled towards its glass transition temperature, $T_{g}$.

\section{EXPERIMENT}

The dielectric relative permittivity $\epsilon^{\prime}$ and dielectric loss $\epsilon^{\prime \prime}$ were measured in the frequency range $10^{-3} \mathrm{~Hz}$ to $1 \mathrm{MHz}$ using a Solatron-Shlumberger 1255A frequency response analyzer (FRA) interfaced with a Chelsea dielectric impedance isolator between the dielectric cell and the FRA. The dielectric cell used was a miniature tunable parallel plate capacitor of capacitance $\sim 27 \mathrm{pF}$ purchased from Alfred Tronser GmbH, Germany. The cell containing the sample was suspended using a sample rod in the Oxford special Spectrodn20 top loading cryostat. The temperature of the cell was controlled to $\pm 0.1 \mathrm{~K}$ using an Oxford ITC6 temperature controller over a period of up to $8 \mathrm{~h}$. Temperature of the sample was measured by immersing a PT 100 class A sensor in the cell containing the liquid using a Keithley 105A multimeter. The maximum tolerance at $73 \mathrm{~K}$ is given as \pm 0.55 $\mathrm{K}$, which is better than for most commercial thermocouples.

The purity of isoamyl bromide and the solvent 2-methylpentane are described in our previous paper. ${ }^{1}$ The mixtures $25 \%, 50 \%, 75 \%$, all in mol \%, were prepared by accurately weighing the appropriate amounts.

\section{RATE EQUATIONS GOVERNING THE RELAXATION PHENOMENA}

The Arrhenius equation for the temperature dependence of a characteristic relaxation time, $\tau$, is given by

$$
\tau^{-1}=\tau_{0}^{-1} \cdot e^{-\left(E_{A} / k_{B} T\right)} .
$$

The apparent activation energy, $E_{A}$, of a typical relaxation process in phenomenological terms increases significantly while approaching the glass transition region. This may tentatively explain a non-Arrhenius temperature dependence of the relaxation time close to $T_{g}$. The pre-exponential factor involving $\tau_{0}$ can be estimated from the relaxation time $\tau$ of a librational process, with zero barrier height, which does not involve crossing over an energy barrier and for which $E_{A}$ $=0$.

The most widely used empirical law which fits reasonably well the temperature dependence of the relaxation time is the Vogel-Fulcher-Tammann ${ }^{9-11}$ (VFT) equation,

$$
\tau^{-1}=\tau_{0}^{-1} \cdot e^{-\left[B /\left(T-T_{0}\right)\right]} .
$$

This law can be explained in terms of Adam and Gibbs ${ }^{12}$ configurational entropy theory, leading to the following equation:

$$
\tau^{-1}=\tau_{0}^{-1} \cdot e^{-\left(S_{c}^{*} \cdot \Delta \mu / k_{B} T S_{\text {conf }}\right)} .
$$

$S_{C}^{*}$ is the critical configurational entropy and $\Delta \mu$ is the energy barrier for the reorientation of a monomer group. The ratio $S_{C}^{*} \cdot \Delta \mu / k_{B}$ is denoted by a constant $C$. It was suggested $^{13}$ that the configurational entropy, $S_{\text {conf }}$, which is temperature dependent, be replaced by $\Delta S=S_{\text {melt }}-S_{\text {crystal }}$. It is found experimentally that $\Delta S$ decreases as $T$ approaches $T_{g}$, and this gives rise to a significant increase in $\tau$ as $T$ $\rightarrow T_{g}$, thus providing a physical basis of the VFT equation. Johari ${ }^{14}$ has recently shown that $S_{\text {conf }}$ is higher than $\Delta S$ and only an upper limit of $S_{\text {conf }}$ can be estimated by extrapolation of the vibrational entropy of a glass at $T=T_{g}$.

The recent development of the mode-coupling theory (MCT) given by Götze and Sjörgen ${ }^{15}$ has given rise to an equation that fits the data and gives a crossover temperature, $T_{C}$, below which the relaxation time predicted by this equation significantly diverges from the experimental value,

$$
\tau^{-1}=k_{1} \cdot\left(\frac{T-T_{C}}{T_{C}}\right)^{\gamma_{1}}, \quad \gamma_{1}>0,
$$

and Souletie and Bertrand, ${ }^{16}$

$$
\tau^{-1}=k_{2} \cdot\left(\frac{T-T_{C}}{T}\right)^{\gamma_{1}}, \quad \gamma_{1}>0,
$$

where $k_{1}$ and $k_{2}$ are constants. The crossover temperature, $T_{C}$, is some $20 \mathrm{~K}$ above $T_{g}$. The exponent $\gamma_{1}$, in particular, is predicted to lie between 2 and 4 .

In the frequency domain, and except for extremely very high frequencies where the inertial effects dominate, the complex relative permittivity, $\epsilon(\omega)$, as a function of angular frequency, $\omega=2 \pi f$, can be fitted to Havriliak-Negami ${ }^{8}$ (HN) equation,

$$
\epsilon(\omega)=\epsilon_{\infty}+\Delta \epsilon \cdot\left[1+\left(i \omega \tau_{\mathrm{HN}}\right)^{\alpha}\right]^{-\beta} .
$$

$\tau_{\mathrm{HN}}$ denotes the characteristic HN relaxation time. $\epsilon_{\infty}$ and $\Delta \epsilon=\epsilon_{S}-\epsilon_{\infty}$ are the permittivity in the high frequency limit and the dielectric relaxation amplitude, respectively. $\epsilon_{S}$ is the static permittivity. The exponents $0<\alpha, \beta<1$ introduce the required symmetry and asymmetric broadening in the permittivity and loss curves.

The frequency of the relaxation peak was calculated from $^{17}$

$f_{m}=\left(2 \pi \tau_{\mathrm{HN}}\right)^{-1}\left(\sin \left[\frac{\alpha \pi}{2+2 \beta}\right]\right)^{1 / \alpha}\left(\sin \left[\frac{\alpha \beta \pi}{2+2 \beta}\right]\right)^{-1 / \alpha}$,

where the relaxation time, $\tau=1 / 2 \pi f_{m}$.

\section{RESULTS AND DISCUSSION}

Figure 1 show the $\epsilon^{\prime}$ and $\epsilon^{\prime \prime}$ spectra of $75 \mathrm{~mol} \%$ solution of isoamyl bromide in 2-methylpentane at 18 selected temperatures. The spectra at temperatures above $124 \mathrm{~K}$ show lower values of $\epsilon^{\prime}$ and $\epsilon^{\prime \prime}$ than expected on the basis of an increase in temperature alone. This is due to the partial freezing of the sample. For the $75 \mathrm{~mol} \%$ solution, we find that the data, at temperatures others than those of partial freezing, can be resolved in terms of two processes. The data are fitted to two Havrilaik-Negami ${ }^{8}$ terms. The set of parameters for both processes, $\left(\Delta \epsilon_{1}, \Delta \epsilon_{2}\right)$ and $\left(\alpha_{1}, \beta_{1} ; \alpha_{2}, \beta_{2}\right)$ are plotted 


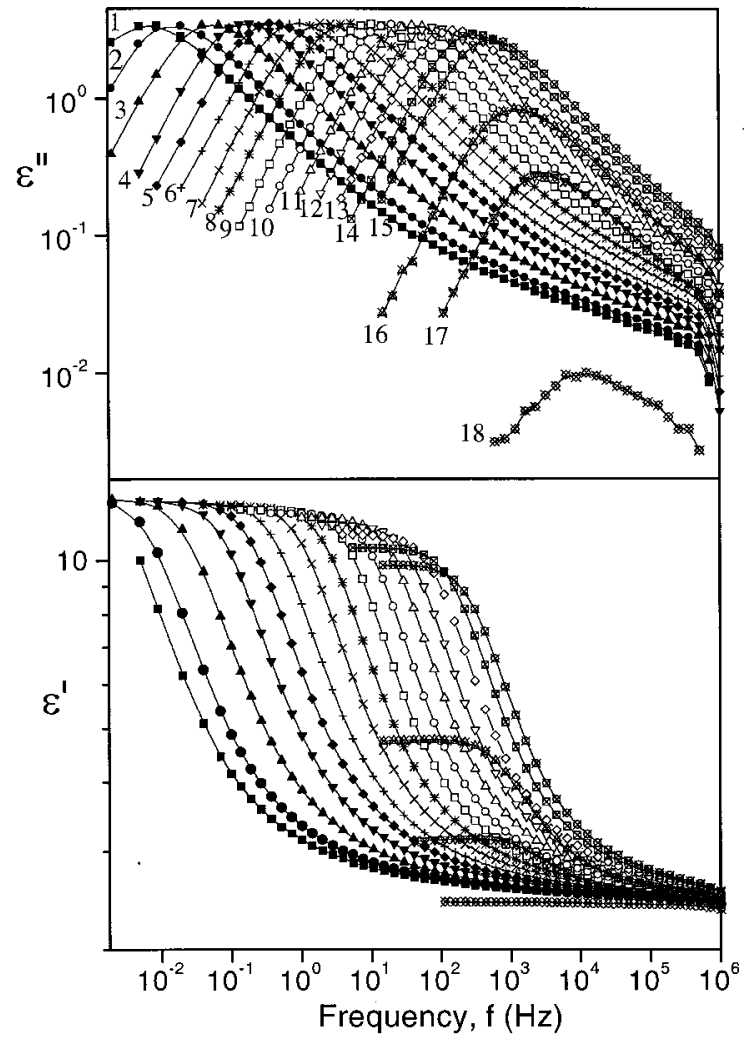

FIG. 1. The $\epsilon^{\prime}$ and $\epsilon^{\prime \prime}$ spectra of $75 \mathrm{~mol} \%$ solution of isoamyl bromide in 2-methylpentane at several temperatures: $1,108.99 ; 2,109.57 ; 3,110.7 ; 4$, $111.92 ; 5,112.83 ; 6,113.77 ; 7,114.72 ; 8,115.64 ; 9,116.67 ; 10,117.64 ; 11$, $118.67 ; 12,119.63 ; 13,120.68 ; 14,121.7 ; 15,122.64 ; 16,123.54 ; 17$, $124.75 ; 18,126.47$.

in Figs. 2 and 3, respectively. It is interesting to note that the low frequency process dominates and its dielectric amplitude is higher by a factor of almost 50 compared to the high frequency process. The average value of $\alpha_{1}$ for the primary process is $0.95 \pm 0.2$ and that of $\beta_{1}$ is 0.58 . This process is found to be $\mathrm{H}-\mathrm{N}$; though it is somewhat closer to Davidson-Cole ${ }^{7}$ formalism, but it is distinguishable only

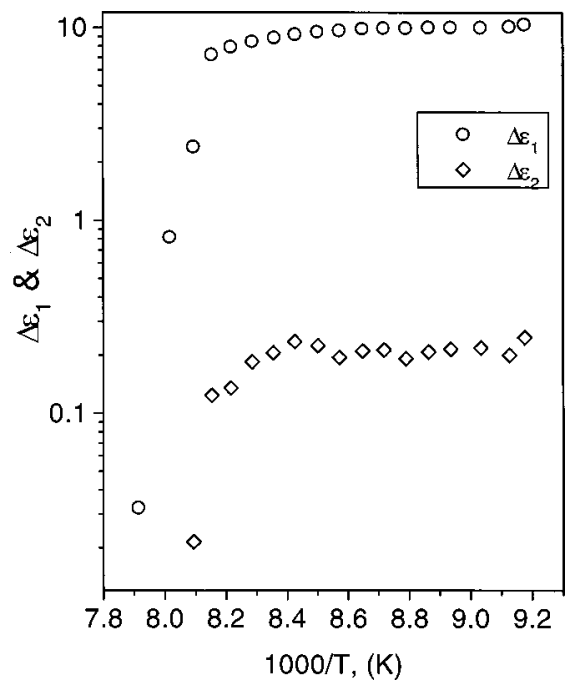

FIG. 2. The plot of the magnitude of relaxation for $75 \mathrm{~mol} \%$ solution of isoamyl bromide in 2-methylpentane against reciprocal temperature.

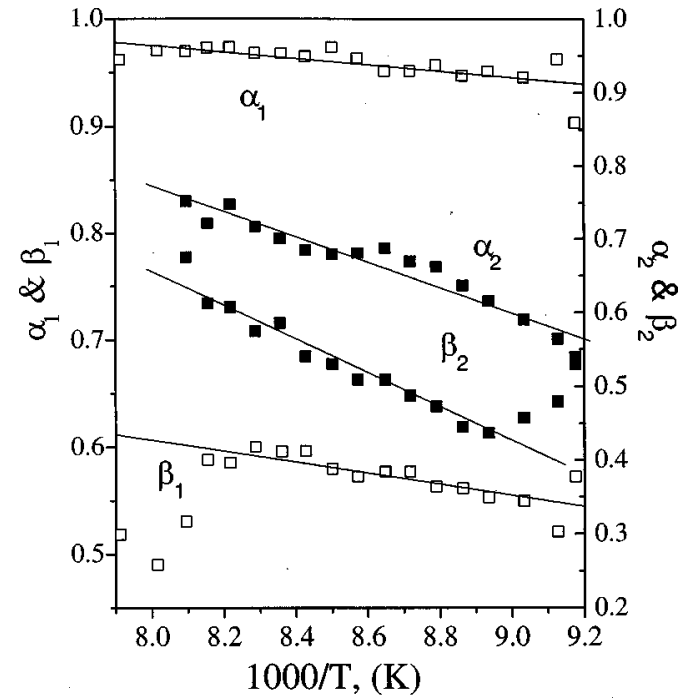

FIG. 3. The plot of the parameters $\alpha$ and $\beta$ for primary and secondary processes against reciprocal temperature for $75 \mathrm{~mol} \%$ solution of isoamyl bromide in 2-methylpentane.

through a fitting procedure on an accurate set of data as being reported here. The second process has clearly both symmetric and asymmetric distribution of relaxation times with $\alpha_{2}$ lying in the range $0.75-0.55$ and decreasing with decreasing temperature, whereas $\beta_{2}$ lies within 0.6 and 0.45 . Frequencies of maximum dielectric loss are calculated using Eq. (7) from the values of the parameters for the two processes that have been calculated by fitting the data. These frequencies are plotted in Fig. 4 as a function of 1000/T. Partial-freezing may also be reflected in the relaxation rate as the point of inflexion is seen at $\sim 124 \mathrm{~K}$ in Fig. 4 . For temperatures excluding those of partial freezing, process 1 is fitted to Arrhenius, VFT, to the mode-coupling theory, ${ }^{15}$ and to the Souletie and Bertrand ${ }^{16}$ equation. The fitting is shown in Fig. 5, and fitting parameters are presented in Table I. The deviations at low temperatures from the Arrhenius law for

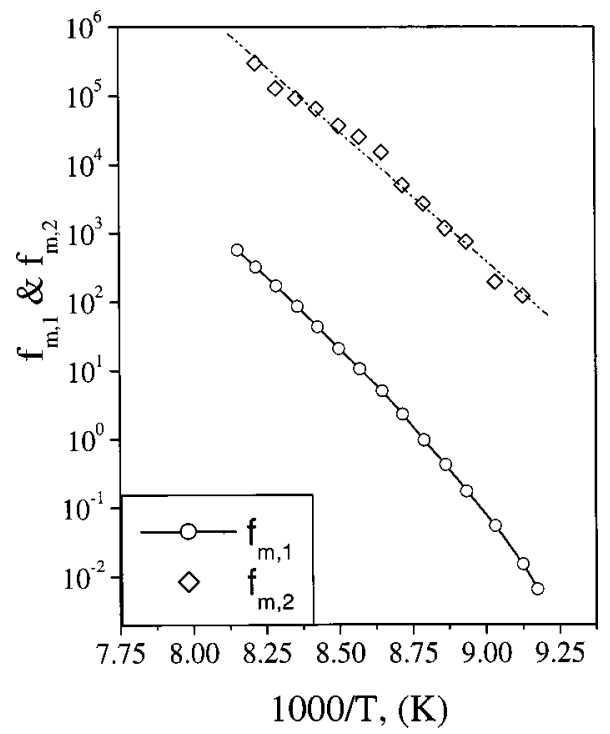

FIG. 4. The plot of $\log f_{m}$ for both processes against reciprocal temperature for $75 \mathrm{~mol} \%$ solution of isoamyl bromide in 2-methylpentane. 


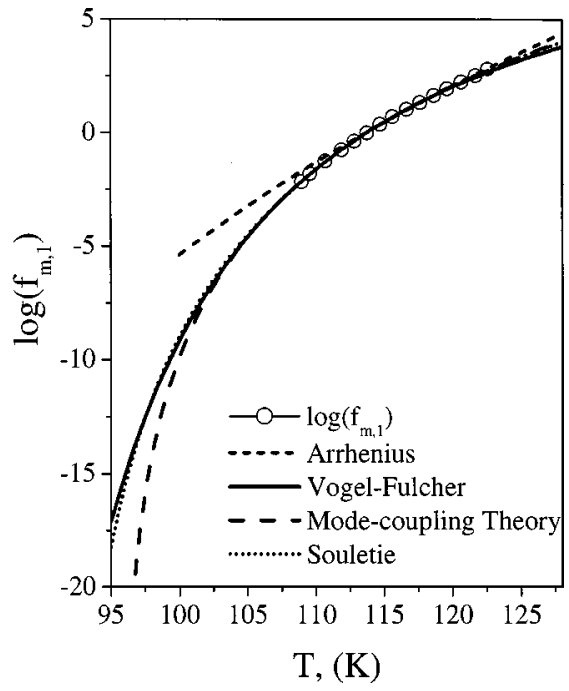

FIG. 5. The plot of $\log f_{m}$ for primary process against temperature in $\mathrm{K}$ for $75 \mathrm{~mol} \%$ solution of isoamyl bromide in 2-methylpentane; and fitting of experimental data to various equations. The equations and parameters of fitting are given in Table I.

the primary process are similar to those already observed. The amplitude of the high frequency or the secondary process is too low to determine whether this process deviates from the Arrhenius law. The two processes tend to merge at a higher temperature some 40-50 K above $T_{g}$. Particularly at low temperatures, the VFT equation describes the temperature dependence of the process extremely well. The mode-coupling theory fits the data at low temperatures and the relaxation time diverges at a temperature of $105 \mathrm{~K}$, some $20 \mathrm{~K}$ above $T_{g}$, in accordance with the prediction of the mode-coupling theory. $T_{g}$ from the dielectric experiments is usually defined as the temperature where the frequency of maximum dielectric loss is $10^{-3} \mathrm{~Hz}$, and the values of $T_{g}$ thus determined for pure isoamyl bromide and its solutions are listed in Table II. In general $T_{g}$ decreases with increasing dilution because the nonpolar solvent has lower $T_{g}$ than the polar solute.

Figure 6 show the $\epsilon^{\prime}$ and $\epsilon^{\prime \prime}$ spectra for $25 \mathrm{~mol} \%$ solution of isoamyl bromide in 2-methylpentane. The striking difference of this spectra from the spectra of pure isoamyl bromide and that of its $75 \mathrm{~mol} \%$ solution is that the spectra are much broader but nevertheless shows a remarkable resemblance to the dielectric spectra of a polymer such as that of poly(venyl acetate). ${ }^{18}$ The spectra in Fig. 6 cannot be resolved into more than a single process and are thus fitted to

TABLE I. The values of different parameters obtained by fitting the various equations to the temperature dependence of the relaxation rate of the primary process for $75 \mathrm{~mol} \%$ solution of isoamyl bromide in 2-methylpentane.

\begin{tabular}{llccc}
\hline \hline & \multicolumn{1}{c}{ Function } & $A$ & $B, \gamma$ & $T_{0}, T_{c}$ \\
\hline Arrhenius & $\log f=A-B / T$ & 39.2 & 4457 & 0 \\
Vogel-Fulcher & $\log f=A-B /\left(T-T_{0}\right)$ & 12.4 & 403 & 81.35 \\
Mode- & $\log f=A+\gamma \log \left[\left(T-T_{c}\right) / T_{c}\right]$ & 11.6 & 16.1 & 95.58 \\
coupling & $\log f=A+\gamma \log \left[\left(T-T_{c}\right) / T\right]$ & 17.3 & 24.3 & 91.74 \\
Souletie &
\end{tabular}

TABLE II. $T_{g}$ for pure isoamyl bromide and the various solutions.

\begin{tabular}{cc}
\hline \hline $\begin{array}{c}\text { Concentration } \\
\text { of IAB (mol \%) }\end{array}$ & $T_{g}(\mathrm{~K})$ \\
\hline 100 & 108.2 \\
75 & 107.2 \\
50 & 95.1 \\
25 & 88 \\
\hline \hline
\end{tabular}

a single $\mathrm{H}-\mathrm{N}$ function; the parameters of the fit are shown in Fig. 7. From Fig. 7, it is interesting to note that an increase in temperature results in the parameter $\alpha$ increasing from a low value of 0.42 close to $T_{g}$ to 0.85 , whereas $\beta$ decreases from almost unity to $\sim 0.6$. This result particularly shows that the symmetric distribution of relaxation times at lower temperatures is gradually transformed to an asymmetric distribution with an increase in temperature. In general at all temperatures $\mathrm{H}-\mathrm{N}$ (Ref. 8) is found to be a better representation of the data than Cole-Cole ${ }^{19}$ or Davidson-Cole ${ }^{7}$ representation alone. Figure 8 shows the frequency of maximum dielectric loss as a function of temperature for the various fits. The parameters of the fitting are presented in Table III. The parameters of fit show a striking difference from those at $75 \%$. In particular, the VFT, $\mathrm{A}=\tau_{0}^{-1}$, parameter for the $25 \%$ solution here is 17.7 as compared to 12.4 for $75 \%$. Values of the parameters, in general, are dependent on the range of temperatures used to fit the data. The parameter, $A$, is much higher than a normally extrapolated value of $\sim 12$, frequency in the THz. This may show that for $25 \%$ solution, the curve

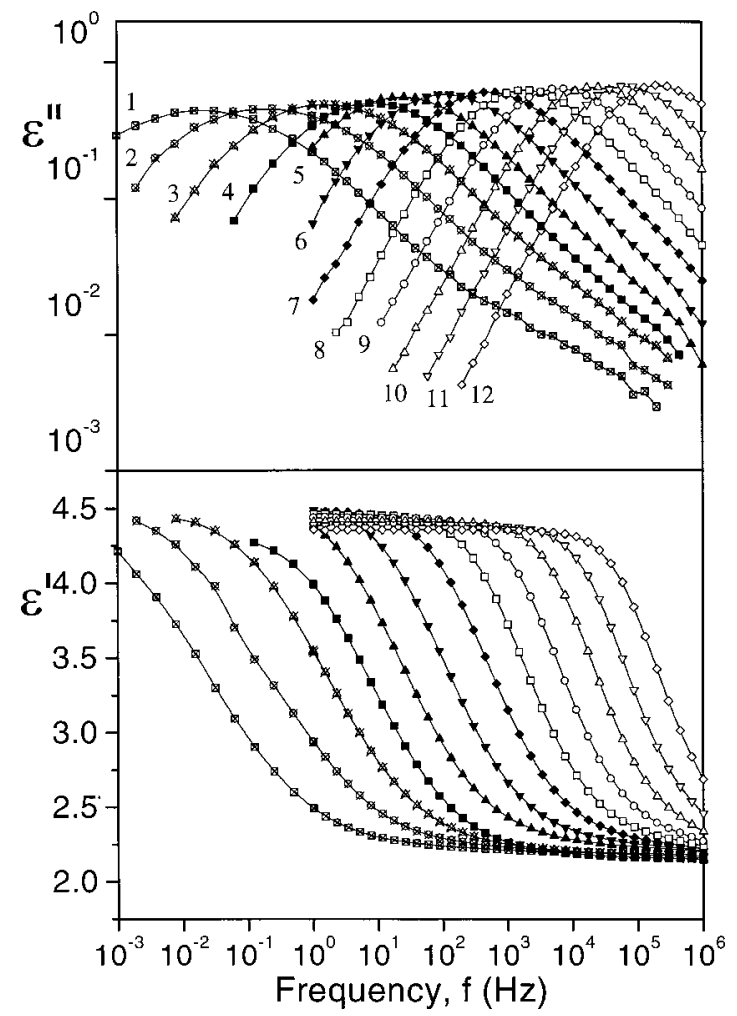

FIG. 6. The $\epsilon^{\prime}$ and $\epsilon^{\prime \prime}$ spectra of $25 \mathrm{~mol} \%$ solution of isoamyl bromide in 2-methylpentane at several temperatures: $1,91.73 ; 2,93.99 ; 3,96.1 ; 4$, $98.87 ; 5,99.29 ; 6,101.39 ; 7,103.62 ; 8,105.64 ; 9,107.71 ; 10,109.92 ; 11$, $112.18 ; 12,114.64$. 


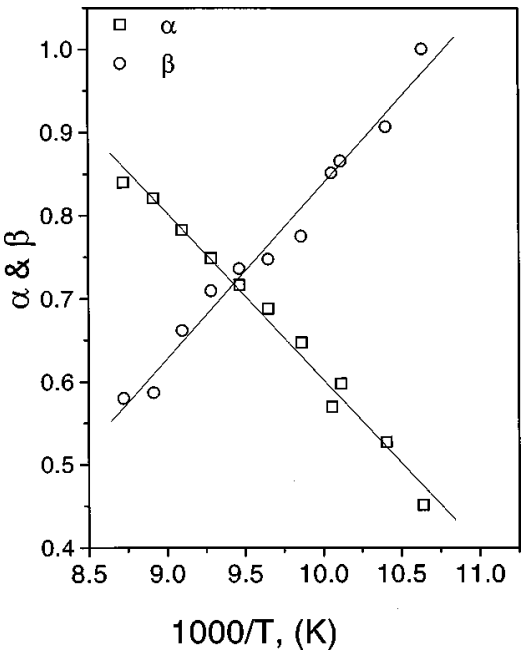

FIG. 7. The plot of the parameters $\alpha$ and $\beta$ against reciprocal temperature for $25 \mathrm{~mol} \%$ solution of isoamyl bromide in 2-methylpentane.

at higher temperatures and consequently higher frequencies must depart significantly from the Arrhenius behavior and thus be considerable bent to reduce $A$ from 17.7 to $\sim 12$. The exponent of the mode-coupling theory is found to be 17.2, which is much greater than predicted by the theory of it lying between 2 and 4 .

In Fig. 9, we show a plot of $\log \epsilon^{\prime \prime}$ vs $\log f$. The data at the low and high frequencies extrapolates to straight lines, the slopes $m$ and $n$ are calculated from fits at low and high frequencies, respectively, shown by chained lines, in Fig. 9. These scaling parameters related to the parameters of the $\mathrm{H}-\mathrm{N}$ equation can be found as follows:

Equation (7) can be written as

$$
\boldsymbol{\epsilon}(\omega)-\boldsymbol{\epsilon}_{\infty}=\Delta \boldsymbol{\epsilon}\left(1+e^{i \alpha \pi / 2}\left(\omega \tau_{\mathrm{HN}}\right)^{\alpha}\right)^{-\beta} .
$$

For $\omega \tau_{\mathrm{HN}} \ll 1$, the equation approximates to

$$
\epsilon(\omega)-\epsilon_{\infty} \approx \Delta \epsilon\left(1-\beta e^{i \alpha \pi / 2}\left(\omega \tau_{\mathrm{HN}}\right)^{\alpha}\right) .
$$

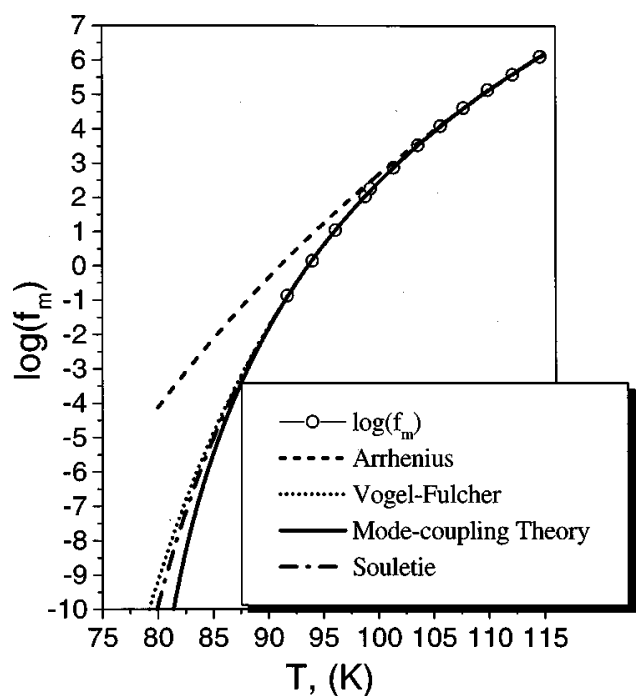

FIG. 8. The plot of $\log f_{m}$ for primary process against temperature in $\mathrm{K}$ for $25 \mathrm{~mol} \%$ solution of isoamyl bromide in 2-methylpentane; and fitting of experimental data to various equations. The equations and parameters of fitting are given in Table III.
TABLE III. The values of different parameters obtained by fitting the various equations to the temperature dependence of the relaxation rate for 25 mol \% solution of isoamyl bromide in 2-methylpentane.

\begin{tabular}{llccc}
\hline \hline & \multicolumn{1}{c}{ Function } & $A$ & $B, \gamma$ & $T_{0}, T_{c}$ \\
\hline Arrhenius & $\log f=A-B / T$ & 29.8 & 2710 & 0 \\
Vogel-Fulcher & $\log f=A-B /\left(T-T_{0}\right)$ & 17.7 & 709 & 53.57 \\
Mode- & $\log f=A+\gamma \log \left[\left(T-T_{c}\right) / T_{c}\right]$ & 11.5 & 17.2 & 77 \\
$\begin{array}{l}\text { coupling } \\
\text { Souletie }\end{array}$ & $\log f=A+\gamma \log \left[\left(T-T_{c}\right) / T\right]$ & 19.4 & 32.6 & 69.9 \\
\hline \hline
\end{tabular}

On equating the imaginary parts, we get

$$
\epsilon^{\prime \prime}(\omega) \approx \Delta \epsilon \beta\left(\omega \tau_{\mathrm{HN}}\right)^{\alpha} \sin \frac{\alpha \pi}{2} .
$$

The plot of $\log \left(\epsilon^{\prime \prime}(f)\right)$ vs $\log f$ at low frequencies gives a straight line with a slope $m=\alpha$.

For high frequencies, $\omega \tau_{\mathrm{HN}} \gg 1$, Eq. (6) yields

$$
\epsilon(\omega)-\epsilon_{\infty} \approx \Delta \epsilon e^{-i \alpha \beta \pi / 2}\left(\omega \tau_{\mathrm{HN}}\right)^{-\alpha \beta} .
$$

On equating the imaginary parts, we get

$$
\epsilon^{\prime \prime}(\omega) \approx \Delta \epsilon\left(\omega \tau_{\mathrm{HN}}\right)^{-\alpha \beta} \sin \frac{\alpha \beta \pi}{2} .
$$

The plot of $\log \left(\epsilon^{\prime \prime}(f)\right)$ vs $\log f$ at high frequencies gives a straight line with a negative slope of magnitude $n=\alpha \beta$.

It may be stated that the scaling parameters $m$ and $n$ are related to those of the "universal relaxation law" given by Jonscher, ${ }^{20} m_{J}$ and $n_{J}$ (both of these being positive numbers) as follows:

$$
m_{J}=m, \quad \text { and } \quad n_{J}=1-n .
$$

The "universal relaxation law" ${ }^{20}$ is therefore only a special case of the Havriliak and Negami ${ }^{8}$ equation and represents

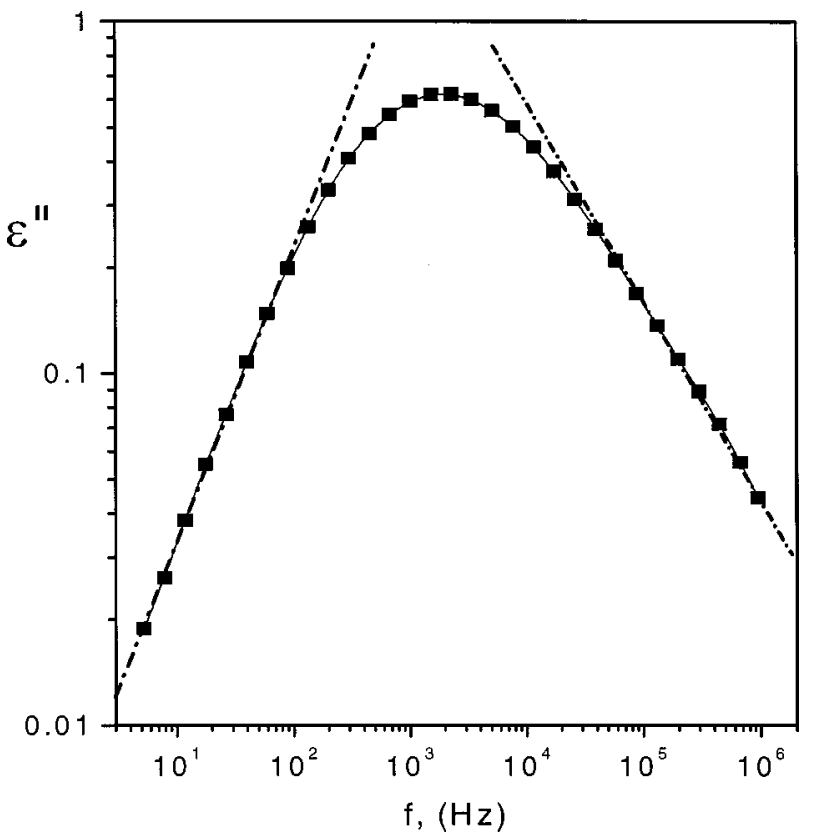

FIG. 9. Example of the $\epsilon^{\prime \prime}$ spectra of $25 \mathrm{~mol} \%$ solution of isoamyl bromide in 2-methylpentane at $T=103.62 \mathrm{~K}$, and fitting of low and high frequency slopes. Fitting parameters for various temperatures are presented in Table III. 


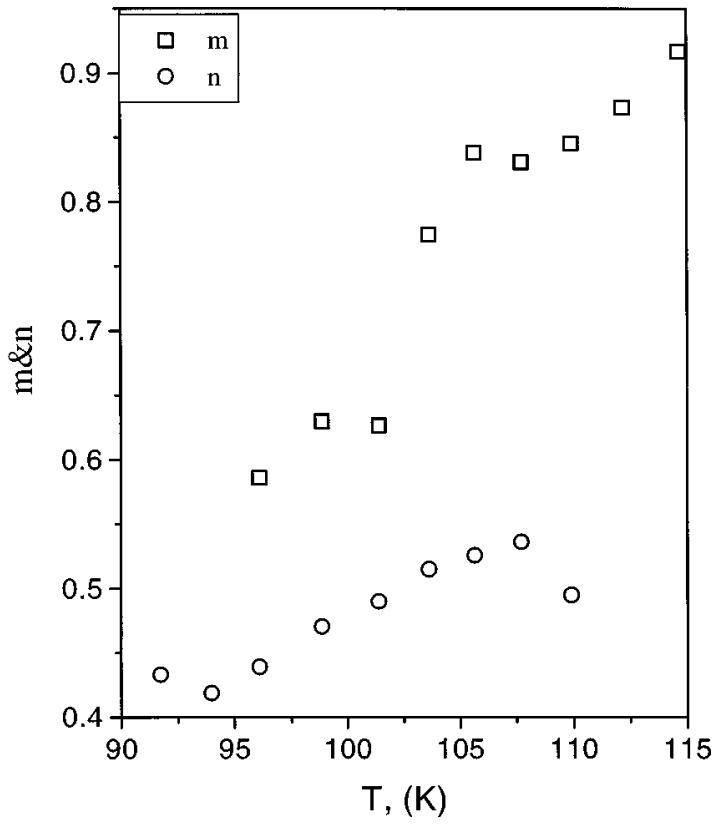

FIG. 10. Temperature dependence of fitting parameters of $m$ and $n$ for 25 mol \% solution of isoamyl bromide in 2-methylpentane.

data in the low and high frequency limits. Values of the scaling parameters $m_{J}$ and $n_{J}$ are dependent on the system ${ }^{21}$ and the mechanism of the process whether it is relaxational or hopping of ions/charges. The latter may also exhibit Debye-type behavior ${ }^{22}$ if the barrier height is much greater than $k_{B} T$.

Except for the extremely high frequencies where the inertial effects dominate, we note that Eqs. (10) and (12) yield power laws in dielectric loss of the form,

$$
\begin{aligned}
& \epsilon^{\prime \prime} \propto \omega^{\alpha} \text { for } \omega \ll \tau_{\mathrm{HN}}^{-1}, \\
& \epsilon^{\prime \prime} \propto \omega^{-\alpha \beta} \text { for } \omega \gg \tau_{\mathrm{HN}}^{-1} .
\end{aligned}
$$

Figure 10 shows a plot of the parameters $m$ and $n$ as a function of temperature. In the temperature range given, $n$ is found to be much lower than unity. We find that whereas both $m$ and $n$ decrease with a decrease in temperature, however the decrease in $m$ with temperature in the range given is found to be much more dramatic. The dynamics at high frequencies reflected in $n \ll 1$ is found to be significantly affected at low temperatures, especially when the system approaches $T_{g}$ from a much higher temperature where $n \leqslant 1$. Two sets of parameters $(m, n)$ and $(\alpha, \beta)$ found by different fitting procedures of the data on $25 \mathrm{~mol} \%$ solution are listed in Table IV. There is found to be a good correspondence between $m=\alpha$ and $n=\alpha \beta$ within the errors of the different fitting procedures. It may be remarked, however, that the procedure of finding $\alpha$, and $\beta$ is much more refined than that of $m$ and $n$, since the latter are the extrapolations of the slopes at low and high frequencies, respectively.

\section{A. Analysis in the time-domain}

In the time-domain, the nonexponential patterns with which equilibrium is restored, following a perturbation, is given by Kohlrausch ${ }^{23}$ stretched exponential law,
TABLE IV. Parameters of fitting of the low and high frequency slopes of dielectric loss spectra of $25 \mathrm{~mol} \%$ solution of isoamyl bromide in 2-methylpentane and $\mathrm{H}-\mathrm{N} \alpha$ and $\beta$.

\begin{tabular}{clllll}
\hline \hline$T(\mathrm{~K})$ & $m$ & \multicolumn{1}{c}{$n$} & $\alpha$ & $\beta$ & $\alpha \beta$ \\
\hline 91.7 & & 0.433 & & & \\
94 & & 0.419 & 0.451 & 1 & 0.451 \\
96.1 & 0.586 & 0.439 & 0.527 & 0.906 & 0.477 \\
98.9 & 0.63 & 0.47 & 0.597 & 0.865 & 0.517 \\
101.4 & 0.626 & 0.49 & 0.647 & 0.774 & 0.501 \\
103.6 & 0.774 & 0.514 & 0.687 & 0.747 & 0.513 \\
105.6 & 0.838 & 0.525 & 0.716 & 0.735 & 0.527 \\
107.7 & 0.831 & 0.536 & 0.748 & 0.709 & 0.531 \\
109.9 & 0.845 & 0.494 & 0.783 & 0.661 & 0.517 \\
112.2 & 0.873 & & 0.821 & 0.586 & 0.481 \\
114.6 & 0.917 & & 0.84 & 0.58 & 0.487 \\
\hline \hline
\end{tabular}

$$
\phi(t)=e^{-(t / \tau)^{\gamma}}, \quad 0<\gamma<1 .
$$

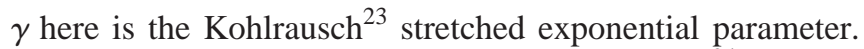
The stretched exponential was used by Douglass ${ }^{24}$ in 1963 for stress relaxation in glasses but applied to dielectrics for the first time by William and Watts ${ }^{25}$ in 1970 and in the literature is termed as $\beta_{\mathrm{KWw}}$. The departure of $\gamma$ from unity is a measure of the nonexponential character of the relaxation function. Numerical Fourier transformation of the stretched exponential law gives a broadened and skewed loss peak in the dielectric loss curve relative to the Debye behavior. There is no direct relationship between the stretched exponential in the time domain and the Havriliak-Negami ${ }^{8}$ in the frequency domain, since Havriliak-Negami ${ }^{8}$ is not a direct Fourier transform of the stretched exponential function (Figs. 11-13).

Since the data on $25 \mathrm{~mol} \%$ solution are shown to fit the $\mathrm{H}-\mathrm{N}$ (Ref. 8) equation extremely well, it is reasonable to conclude that the Havriliak-Negami ${ }^{8}$ equation is a good rep-

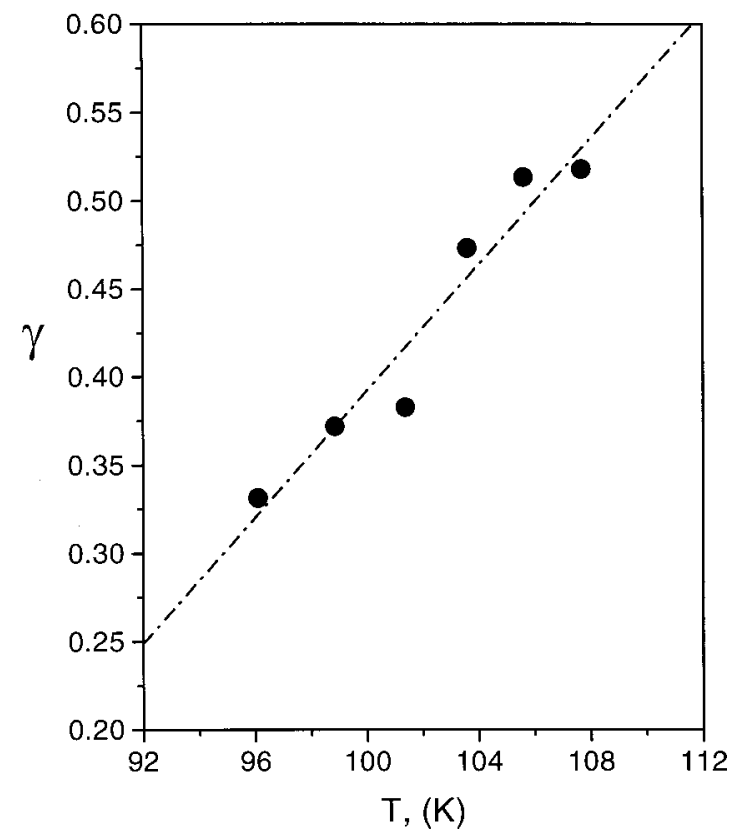

FIG. 11. The temperature dependence of the KWW stretched exponent $\gamma$ on temperature for $25 \mathrm{~mol} \%$ solution as above. 


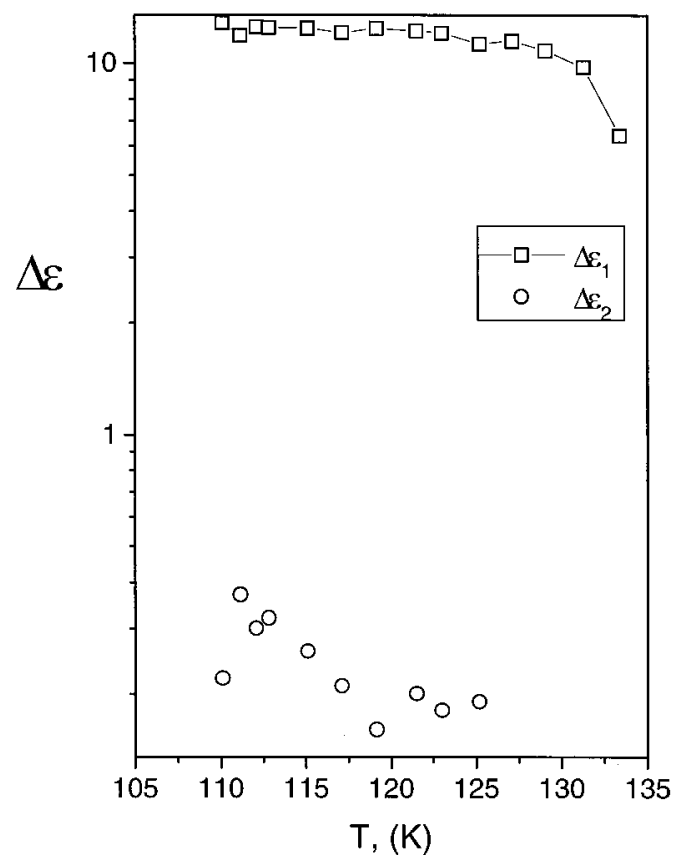

FIG. 12. The plot of the dielectric amplitudes for pure isoamyl bromide against temperature in $\mathrm{K}$.

resentation of the data of some low molecular weight glassforming systems. ${ }^{17,21,26}$ Alvarez et al. ${ }^{27}$ have obtained an empirical connection between the analysis in the time-and the frequency domains. They established the relationship by simulating the data in the time-domain by letting $\beta_{\mathrm{KWW}}(=\gamma$ here), to lie in the range $0.95-0.1$, for times such that the ratio $t / \tau$ lies in between $10^{-3}-10$. This was then numerically Fourier transformed to produce the frequency depen-

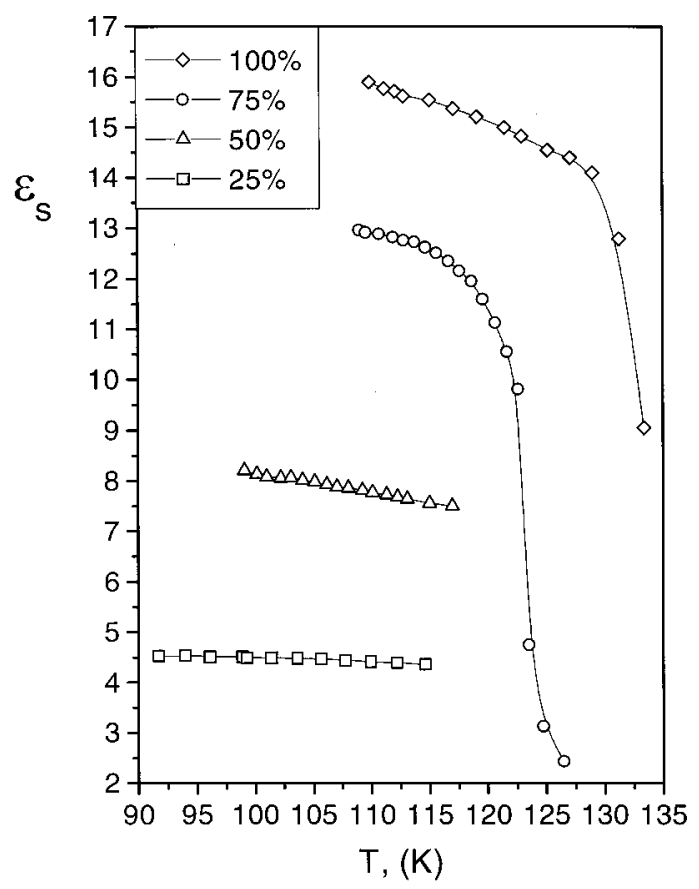

FIG. 13. The $\epsilon_{S}$ of the primary process for pure isoamyl bromide and its 75 , 50 , and $25 \mathrm{~mol} \%$ solutions in 2-methylpentane against the temperature.

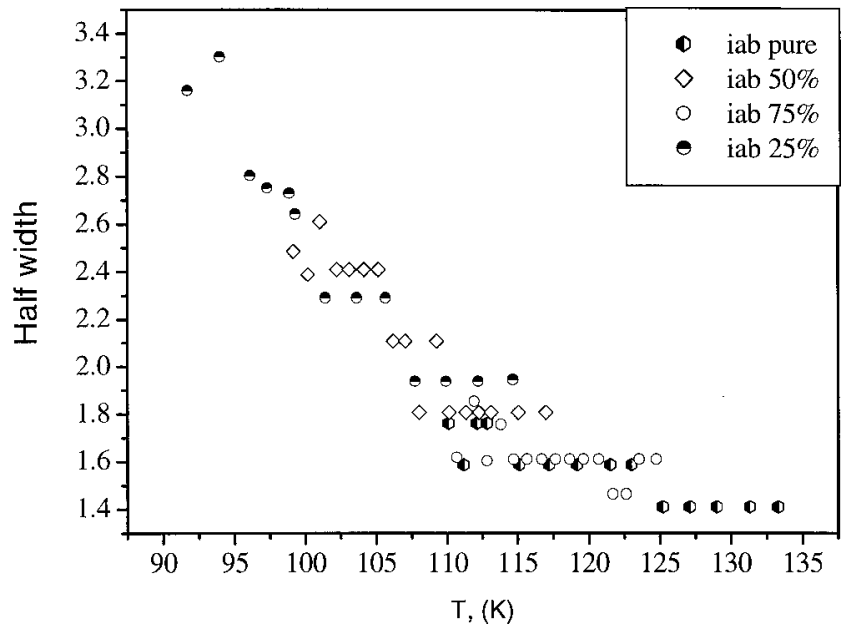

FIG. 14. Dependence of the half-width of the dielectric spectra for different concentrations of isoamyl bromide; half-width of the Debye process $=1.14$ decades .

dence of the spectra and the data fitted to the Havriliak-Negami ${ }^{8}$ equation and $\alpha$ and $\beta$ thus computed. The following empirical equation was found:

$$
\alpha \beta=\gamma^{1.23} \text {. }
$$

This would imply that both $\alpha$ and $\beta$ and in turn the low and high frequency slopes are determined by $\gamma$ as shown by Moynihan et al. ${ }^{28}$ The stretched exponent, $\gamma$, is calculated from $\alpha$ and $\beta$ and is plotted in Fig. 11 as a function of temperature. The plot follows the equation, $\gamma \approx a\left(T-T_{0}\right)$, with $a=0.0179$ and $T_{0}=78.1 \mathrm{~K}$. This in turn will lead to the VFT dependence of the dielectric relaxation time as suggested by Nagel and Dixon. ${ }^{29}$ Lunkenheimer et al. ${ }^{30}$ have found a similar behavior of the stretched exponent with temperature for glycerol, though they did not show any fitting of the data. It may be remarked that $T_{0}$ agrees with that using the mode-coupling theory better than the VFT, however this need to be investigated in detail in future.

The width of the permittivity curves increases with dilution of isoamyl bromide by 2-methylpentane. This is reflected in an increase in the nonexponential character of the relaxation function measured by the parameters $\alpha, \beta$, and the Kohlrausch $\gamma$, and the scaling parameters $m$ and $n$. Figure 14 shows the half-width (HW) of the dielectric loss curves, as a function of temperature for different concentrations. There is found to be a significant increase in the half-width with both dilution and also a reduction in temperature. The dilution through a reduction in the dipole-dipole interaction energy seems to break the cooperativity of polar molecules in the liquid and thus increases the non-exponential character of the relaxation function. Pure isoamyl bromide already shows the nonexponential relaxation character, since the molecules are not linked through any intermolecular forces like those in a normal ${ }^{31}$ and some branched alcohols where the primary process is largely exponential ${ }^{32}$ due to a cooperative process. This is also supported by the results of Glasser et al. ${ }^{33}$ who found that normal alcohols, which in pure state at room temperature give rise to an exponential relaxation dynamics, lead to a wider distribution of the dielectric relaxation times when 
dissolved in a nonpolar solvent. A reduction in the cooperativity thus gives rise to the structural and consequently to the dynamic heterogeneity. ${ }^{34-36}$ The experiments by Weeks et al. ${ }^{37}$ using $3 \mathrm{D}$ confocal microscopy on the colloids has recently shown the existence of a large number of clusters in a liquid. These clusters involve only approximately $5 \%$ of the particles, which themselves are distributed in a number of clusters. The particles in a cluster move together. The remaining $95 \%$ of the particles are relatively static. As the glass transition is approached, the typical size of a cluster increases. A increase in dilution increases the number and a distribution in the size of the clusters and in consequence increases the nonexponential relaxation dynamics. The results given here may therefore indirectly support the premise that loss of cooperativity and an increase in heterogeneity are the primary causes of the nonexponential relaxation dynamics.

\section{B. Johari-Goldstein relaxation}

The 75 mol\% solution of isoamyl bromide in 2-methylpentane clearly exhibits a secondary relaxation process. The dielectric amplitudes of primary and secondary processes $\Delta \epsilon_{1}$ and $\Delta \epsilon_{2}$ are shown in Fig. 2. As already stated, the dielectric amplitude of the higher frequency process is lower by a factor of 50 and is slightly dependent on temperature except close to the freezing temperature $T_{f}$, where $\Delta \epsilon_{2}$ amplitude suddenly drops as does $\Delta \epsilon_{1}$. This observation of secondary process being observed is more marked than for pure isoamyl bromide shown in Fig. 12. Since isoamyl bromide liquid is nonhydrogen bonded and also the rotations around the $\mathrm{C}-\mathrm{C}$ bond of the molecule do not give rise to a change in the dipole moment, the higher frequency process can only be assigned to the Johari-Goldstein ${ }^{38,39}$ type of a relaxation process. The Johari-Goldstein ${ }^{38,39}$ process is exhibited by the isolated dipolar molecules constrained in the voids of the domains/ clusters. The primary process is caused by the collective rotational/torsional motion of the majority of molecules that lie within the domains of the system. The relative predominance of the secondary process is therefore dependent on the system and on the rate of cooling. The existence of this process is dependent on whether the system allows the formation of voids of sufficiently large size where the dipolar molecules have the possibility of being trapped and of executing torsional motion. In most glass-forming low molecular weight systems, these voids are naturally formed as the system is cooled towards $T_{g}$. This is the main reason why the secondary relaxation in our view is a universal or near universal feature ${ }^{40}$ of the glassy state. In which case, the relative dielectric amplitude of this process compared to the primary process depends on the number density of these voids and on the availability of single dipolar molecules within these voids. Our results show that a small dilution of isoamyl bromide with 2-methylpentane aids in the formation of these voids, which is quite plausible since the solvent molecules tend to break these clusters or domains. However an increase in the concentration of 2-methylpentane tends not only to reduce the size and increase the number of these clusters of polar molecules but also makes these clusters and a small number of isolated polar molecules to act as independent set of relaxors in a very viscous media like those in an amorphous solid. This is reflected by the increased nonexponential behavior of the relaxation time of the main process for 50 and $25 \mathrm{~mol} \%$ solutions. In such cases, it is not easy to separate the primary and secondary processes. For $75 \mathrm{~mol} \%$ solution however, the two processes are not seen to merge for $T>T_{g}$ due to a restricted range of temperatures investigated, but when extrapolated these curves merge at $\sim 145 \mathrm{~K}$, however Fig. 4 of Ref. 1 clearly shows that the two processes merging for $T>T_{g}$. The merger occurs mainly because at increased temperature, the distinction between the primary and the secondary processes disappears. It may be remarked here that some polymers also show the secondary or $\beta$ relaxation but the mechanism for that process is due to the torsional motion of the segments of the polymer, which manifestly is different from that of the Johari-Goldstein process.

\section{Temperature dependence of $\epsilon_{S}$}

The static permittivity, $\epsilon_{S}$, for $75 \%$ and $25 \%$ is shown as a function of the temperature in Fig. 13 and included are also the plots of $\epsilon_{S}$ for pure isoamyl bromide and $50 \mathrm{~mol} \%$ solutions. The extrapolated values of $\epsilon_{S}$ at $110 \mathrm{~K}$ for pure isoamyl bromide, $75 \%, 50 \%, 25 \%$, respectively, are 15.81 , $12.95,7.71$, and 4.38 , respectively. $\epsilon_{S}$ for 2-methylpentane is 2.094. The plot of $\epsilon_{S}$ vs mole fraction of the solute (not given) shows an almost linear increase in $\epsilon_{S}$. The extrapolated densities from the measurements carried out by Denny and Ring ${ }^{6}$ have been obtained. For pure isoamyl bromide and its $75 \%, 50 \%, 25 \%$ solutions at $110 \mathrm{~K}$, these are $1.44,1.26$, 1.10 , and $0.95 \mathrm{~g} / \mathrm{cm}^{3}$. The extrapolated density for 2-methyl pentane is found to be $0.78 \mathrm{~g} / \mathrm{cm}^{3}$. The Kirkwood correlation factor, ${ }^{41} \mathrm{~g}$, for different concentrations at $110 \mathrm{~K}$ was calculated, and found to be $g=0.7 \pm 0.1$ within the concentration range investigated. This shows that antiparallel association is present for concentrations of $25 \%$ and above. In the absence of hydrogen-bonding, the antiparallel association seems to be derived from the dipole-dipole interactions and the steric factors concerned with the packing of the molecules in the condensed state. This antiparallel association is not found to be significantly altered at low temperatures by dilution with 2-methylpentane at or above $25 \%$ of isoamyl bromide.

\section{CONCLUSIONS}

Nonhydrogen bonded isoamyl bromide and its solutions in a nonassociated nonpolar solvent 2-methylpentane show nonexponential relaxation dynamics and the increase in the nonexponential character is followed by dilution with 2-methylpentane. This is shown by an increase in the halfwidth of the dielectric loss curves, and a reduction in the stretched exponential exponent, the Kaulrausch parameter, $\gamma$. For these mixtures, Havriliak-Negami equation in the frequency domain is shown to fit the data extremely well and $\gamma$ is estimated as a function of temperature. This is shown to lead to the equation, which in turn leads to the VFT equation for the relaxation peak frequency as a function of temperature. The secondary relaxation in pure isoamyl bromide and its $75 \mathrm{~mol} \%$ solution is interpreted in terms of the Johari- 
Goldstein process and this process is not observed with increase in dilution, for which an explanation is given. A reduction in the cooperativity among the polar molecules and consequently an increase in the heterogeneity is found to be the main cause of the non-exponential relaxation dynamics.

\section{ACKNOWLEDGMENTS}

We thank the American Petroleum Research Fund of the American Chemical Society, PRF \#33221-AC7, for funding this work. We are extremely grateful to Professor G. P. Johari and Professor B. K. P. Scaife for the stimulating discussions.

${ }^{1}$ O. E. Kalinovskaya and J. K. Vij, J. Chem. Phys. 111, 10979 (1999).

${ }^{2}$ S. H. Glarum, J. Chem. Phys. 33, 639 (1960).

${ }^{3}$ D. J. Denny, J. Chem. Phys. 27, 259 (1957).

${ }^{4}$ J. G. Berberian and R. H. Cole, J. Am. Chem. Soc. 90, 3100 (1968).

${ }^{5}$ J. G. Berberian, IEEE Trans. Electr. Insul. EI-20, 931 (1985).

${ }^{6}$ D. J. Denny and J. W. Ring, J. Chem. Phys. 44, 4621 (1966).

${ }^{7}$ D. W. Davidson and R. H. Cole, J. Chem. Phys. 18, 1417 (1950); 19, 1484 (1951).

${ }^{8}$ S. Havriliak, Jr. and S. Negami, J. Polym. Sci., Part C: Polym. Symp. 14, 89 (1966); Polymer 8, 161 (1967).

${ }^{9}$ H. Vogel, Phys. Z. 22, 645 (1921).

${ }^{10}$ G. S. Fulcher, J. Am. Ceram. Soc. 8, 339 (1923).

${ }^{11}$ G. Tammann and W. Hesse, Z. Anorg. Allg. Chem. 156, 245 (1926).

${ }^{12}$ G. Adam and J. H. Gibbs, J. Chem. Phys. 43, 139 (1965).

${ }^{13}$ R. Richert and C. A. Angell, J. Chem. Phys. 108, 9016 (1998).

${ }^{14}$ G. P. Johari, J. Chem. Phys. 112, 7518 (2000).

${ }^{15}$ W. Götze and L. Sjörgren, Rep. Prog. Phys. 55, 241 (1992).

${ }^{16}$ J. Souletie and D. Bertrand, J. Phys. I 1, 1627 (1991).

${ }^{17}$ F. Stickel, E. W. Fischer, and R. Richert, J. Chem. Phys. 102, 6251 (1995).

${ }^{18}$ S. Mashimo, R. Nozaki, S. Yagihara, and S. Takeishi, J. Chem. Phys. 77, 6259 (1982).
${ }^{19}$ K. S. Cole and R. H. Cole, J. Chem. Phys. 9, 341 (1941).

${ }^{20}$ A. K. Jonscher, Universal Relaxation Law (Chelsea Dielectrics, London, 1996), ISBN 0950871125.

${ }^{21}$ A. Schönhals, F. Kremer, and E. Schlosser, Phys. Rev. Lett. 67, 999 (1991).

${ }^{22}$ H. Fröhlich, Theory of Dielectrics (Oxford University Press, Oxford, 1958).

${ }^{23}$ R. Kohlrausch, Pogg. Annal. Phys. 91, 179 (1854).

${ }^{24}$ R. W. Douglass, in Proceedings of the 4th International Congress on Rheology, Providence, RI, 1963, Part 1, edited by E. H. Lee and A. L. Copely (Wiley, New York, 1965), pp. 3-27; Br. J. Appl. Phys. 17, 435 (1966).

${ }^{25}$ G. Williams and D. C. Watts, Trans. Faraday Soc. 66, 80 (1970).

${ }^{26}$ A. Schönhals, F. Kremer, A. Hofmann, E. Fischer, and E. Schlosser, Phys. Rev. Lett. 70, 3459 (1993).

${ }^{27}$ F. Alvarez, A. Alegría, and J. Colmenero, Phys. Rev. B 44, 7306 (1991).

${ }^{28}$ C. T. Moynihan, L. P. Boesch, and N. L. Laberge, Phys. Chem. Glasses 14, 122 (1973).

${ }^{29}$ S. R. Nagel and P. K. Dixon, J. Chem. Phys. 90, 3885 (1989).

${ }^{30}$ P. Lunkenheimer, A. Pimenov, B. Schiener, R. Böhmer, and A. Loidl, Europhys. Lett. 33, 611 (1996).

${ }^{31}$ J. K. Vij, C. J. Reid, and M. W. Evans, Mol. Phys. 50, 935 (1983); J. K. Vij, W. G. Scaife, and J. H. Calderwood, J. Phys. D 14, 733 (1981).

${ }^{32}$ O. E. Kalinovskaya and J. K. Vij, J. Chem. Phys. 112, 3262 (2000).

${ }^{33}$ L. Glasser, J. Crossley, and C. P. Smyth, J. Chem. Phys. 57, 3977 (1972).

${ }^{34}$ R. Böhmer, R. V. Chamberlin, G. Diezemann et al., J. Non-Cryst. Solids 235-237, 1 (1998).

${ }^{35}$ K. Schmidt-Rohr and H. W. Spiess, Phys. Rev. Lett. 66, 2851 (1991).

${ }^{36}$ C. T. Moynihan, J. Non-Cryst. Solids 235-237, 781 (1998).

${ }^{37}$ E. Weeks, J. C. Crocker, A. C. Levitt, A. Schofield, and D. A. Weitz, Science 287, 627 (2000).

${ }^{38}$ G. P. Johari and M. Goldstein, J. Chem. Phys. 53, 2372 (1970).

${ }^{39}$ G. P. Johari, J. Chem. Phys. 58, 1766 (1973).

${ }^{40}$ M. Goldstein, J. Chem. Phys. 51, 3728 (1969).

${ }^{41}$ See, for a detailed analysis, B. K. P. Scaife, Principles of Dielectrics (Clarendon, Oxford, 1998), p. 200. 